\title{
Roxadustat in treating anemia in dialysis patients (ROAD): protocol and rationale of a multicenter prospective observational cohort study
}

Yaling Zhang ${ }^{1+}$, Song Ren ${ }^{1 \dagger}$, Hen Xue ${ }^{2 \dagger}$, Amanda Y Wang ${ }^{3,4,5^{*}+}$, Yang Zou ${ }^{1 \dagger}$, Yanrong Cai ${ }^{6}$, Jingdong He ${ }^{7}$, Xiaoling Yuan ${ }^{8}$, Feifei Jiang ${ }^{9}$, Jinxi Wei ${ }^{10}$, Dongmei Yang ${ }^{11}$, Dong He ${ }^{12}$, Shide Hu ${ }^{13}$, Min Lei ${ }^{14}$, Fei Deng ${ }^{1}$, Jin Chen ${ }^{1}$, Xia Wang ${ }^{15}$, Qiang He ${ }^{1 *}$, Guisen $\mathrm{Li}^{1^{*}}$ and Daqing Hong ${ }^{1 *}$

\begin{abstract}
Background: Roxadustat has been shown effective in treating patients with anemia due to chronic kidney disease. However, its long-term effect on clinical outcomes and socioeconomic burden and safety remains unclear.

Methods/Design: This is a multicenter, prospective, longitudinal observational cohort study assessing if Roxadustat improves prognosis in dialysis patients. Primary outcomes will be major adverse cardiovascular events (MACE), defined as composites of cardiovascular death, myocardial infarction, cerebral infarction, hospitalization because of heart failure; all-cause mortality, and annual economic costs in two years. The data will be collected via Research electronic data capture (REDCap) based database as well as software-based dialysis registry of Sichuan province. The primary outcomes for the ROAD study participants will be compared with those in the dialysis registry cohort. Data at baseline and study follow up will also be compared to assess the association between Roxadustat and long-term clinical outcomes.
\end{abstract}

Discussion: The main objective of this study is to the assess long-term association of Roxadustat on MACE, allcause mortality, socio-economic burden, safety in dialysis patients, which will provide guidance for designing further large randomized controlled trials to investigate this clinic question.

Study registration: The study has been registered in Chinese Clinical Trials Registry (ROAD, ROxadustat in treating Anemia in Dialysis patients, registration number ChiCTR1900025765) and provincial observational cohort database (Renal disEAse observational CoHort database, REACH, ChiCTR1900024926), registered 07 September 2019, http:// www.chictr.org.cn.

Keywords: Roxadustat renal anemia hemodialysis

\footnotetext{
*Correspondence: 13111865150@163.com; 13111865150@163.com;

guisenli@163.com; hongdaqing11@126.com

${ }^{3}$ The Renal and Metabolic Division, The George Institute for Global Health,

University of New South Wales, Sydney 2042, Australia

${ }^{1}$ Renal Department and Nephrology Institute, Sichuan Provincial People's

Hospital, School of Medicine, University of Electronic Science and

Technology of China, No. 32, West 2nd Duan, 1st Circle Road, Qingyang

District, Chengdu, Sichuan, China

Full list of author information is available at the end of the article
}

(C) The Author(s). 2021 Open Access This article is licensed under a Creative Commons Attribution 4.0 International License, which permits use, sharing, adaptation, distribution and reproduction in any medium or format, as long as you give appropriate credit to the original author(s) and the source, provide a link to the Creative Commons licence, and indicate if changes were made. The images or other third party material in this article are included in the article's Creative Commons licence, unless indicated otherwise in a credit line to the material. If material is not included in the article's Creative Commons licence and your intended use is not permitted by statutory regulation or exceeds the permitted use, you will need to obtain permission directly from the copyright holder. To view a copy of this licence, visit http://creativecommons.org/licenses/by/4.0/ The Creative Commons Public Domain Dedication waiver (http://creativecommons.org/publicdomain/zero/1.0/) applies to the data made available in this article, unless otherwise stated in a credit line to the data. 


\section{Background}

The incidence and prevalence of Chronic kidney disease (CKD)is increasing globally which affects about $10 \%$ of people worldwide [1]. In China, the estimated number of people with CKD is 120 million [2], among which 2\% will progress to end-stage renal disease (ESRD).

Renal anemia is one of the common complications of CKD especially ESRD, which is predominantly due to the reduction in erythropoietin (EPO) production by the kidney. Renal anemia can occur in any stages of CKD, but its prevalence and severity increases with the progression of CKD [3]. It is reported that more than $90 \%$ of dialysis patients have renal anemia [4], which is associated with increased risk of cardiovascular events [5, 6] and all-cause mortality [7].

Erythropoiesis-stimulating agents (ESA) are commonly used in the treatment of renal anemia. It can not only improve hemoglobin levels, but also cardiovascular outcomes in CKD patients. It can also delay the progression of chronic kidney disease, reduce hospitalization, allcause mortality, and improve the quality of life of CKD patients [8]. The DOPPS study found that for every $10 \mathrm{~g} / \mathrm{L}$ increase of hemoglobin (HGB), the risk of death and hospitalization decreased by $5 \%$ and $4 \%$ respectively [9]. However, the use of ESA also carries adverse events. For example, high dose of ESA is associated with increased risk of cardiovascular events and mortality, and regardless of HGB levels [10], demonstrated by the CHOICE study. In addition, ESA resistance can occur in some patients, resulting in treatment failure.

In recent years, hypoxia inducible factor (HIF) related medications have been tested in the treatment of renal anemia in a number of trials. HIF can increase the production of endogenous EPO by inhibiting the activity of prolyl hydroxylase. Roxadustat, as a HIF prolyl hydroxylase inhibitor (HIF-PHI), has been completed Clinical trials in several countries. The most recent randomized, multicenter, doubleblind clinical studies, done in China, have found that Roxadustat significantly improved the hemoglobin level in CKD patients on and not on dialysis $[8,11]$. Other studies also showed Roxadustat can effectively correct renal anemia, and reduce the requirements for blood transfusion and intravenous iron supplementation $[3,12,13]$.

Despite the proven short-term benefits of Roxadustat in CKD patients, its long-term effect on clinical outcomes and socio-economic burden remains uncertain. Therefore, this study aims to perform a prospective longitudinal cohort study assessing the long-term effect of Roxadustat on cardiovascular events and mortality, as well as economic costs in dialysis patients with renal anemia.

\section{Methods/Design}

\section{Study design}

This is a multicenter, prospective, longitudinal cohort study. It has been registered in Chinese Clinical Trials Registry
(ROAD, ROxadustat in treating Anemia in Dialysis patients, registration number ChiCTR1900025765), provincial observational cohort database (Renal disEAse observational CoHort database, REACH, ChiCTR1900024926).

\section{Study population}

The participants included in the ROAD study will be patients with renal anemia in the context of end-stage kidney disease on dialysis (both hemodialysis and peritoneal dialysis), and on Roxadustat treatment who consent to the study. The de-identified data from the provincial dialysis registry of dialysis patients with renal anemia will be serves as a control group. The study was approved by the Institutional Review Board at Sichuan Provincial People's Hospital (2019-196). The informed consent obtained from study participants will be written.

Inclusion criteria include:(1)Age 18-90 years; (2)Endstage kidney disease patients who are receiving maintenance hemodialysis or peritoneal dialysis;(3Dry weight 45-160 kg; (4) Renal anemia, defined as $\mathrm{HGB}<130 \mathrm{~g} /$ (male), $\mathrm{HGB}<120 \mathrm{~g} / \mathrm{L}$ (female) or $\mathrm{HGB}$ is maintained normal by ESA; (5)willing to participate in the study with informed consent. Exclusion criteria includes:(1)patients with pre-existing malignancy; (2)Known history of hematological disorders including myelodysplastic syndrome, multiple myeloma, hereditary hematologic disease such as thalassemia, sickle cell anemia, pure red cell aplasia, or other known causes for anemia other than CKD or dialysis. (3) Patients who cannot complete the follow-up study.

\section{Study outcomes}

Primary outcomes will be major adverse cardiovascular events (MACE), defined as composite of cardiovascular death, myocardial infarction, cerebral infarction, hospitalization because of heart failure or unstable angina pectoris; all-cause mortality and annually medical economic costs in 2 years.

Secondary outcomes will include: (1)Mean HGB changes from baseline to the 16th week and percentage of patients within HGB treatment target (110-130 g/L). (2) Changes from baseline and variations in the mean HGB level throughout the whole study periods (up to 108 weeks). (3) Changes of serum iron metabolism indexes (Hepcidin, ferritin, transferrin, transferrin saturation, and total iron binding capacity) as well as requirements for intravenous iron replacement from baseline to the end of the study(up to 108 weeks). (4)Changes of blood pressure and antihypertensive medications from baseline to 16th week. (5)Changes of serum lipids profile from baseline to 16th week. (6) Changes of the following laboratory results (collected in the Sichuan provincial dialysis registration system) from baseline to the end of study, dialysis related parameters, dialysis 
adequacy and chronic kidney disease- mineral bone disorder (CKD-MBD) markers (serum phosphorus, calcium, and parathyroid hormone). (7) Mean weekly dosage of Roxadustat to maintain HGB within the treatment target. (8)Adverse events (AE) during the follow-up period, with a focus on drug discontinuation due to AE. (9)Annual economic cost related to hospitalization, outpatient treatments, medical insurance coverage and outof-pocket payments.

\section{Data collection}

The de-identified data were collected in REACH via Research electronic data capture (REDCap [14, 15], hosted at Sichuan Provincial People's Hospital) pre-designed forms from baseline to week 108/2 years. The table with an identification code will be kept within the study site in a secure way. The provision of de-identified medical records will be required for judication for the MACE outcome. Participants will be followed up to 2 years except that they withdraw from the study, have their dialysis type changed, receive kidney transplantation or have lost follow up.

Dialysis data from each study site in the provincial dialysis registry will be collected via a dialysis software provided by the Sichuan Quality Control Center of Renal Disease at the end of the first and second study year.

A questionnaire focusing on medical expense for the participants will be collected at the end of the first and second year of the study.

The target sample size of the ROAD participant is greater than 250 basing on the MACE incidence of at least $20 \%[16,17]$ within 2 years among ROAD and dialysis registry cohort, basing on noninferiority assumption with a marginal difference of 0.02 (10\% of MACE incidence), actual difference of -0.08 (40\% of MACE incidence), $\alpha=0.05$ and $1-\beta=0.8$, allowing a drop rate of $15 \%$, using 'Non-Inferiority Tests for the Difference Between Two Proportions' of PASS software 15.0.5.

\section{Quality control}

The investigators will be trained before initiation of the study. A multi-disciplinary team will provide consultation on adjustment of Roxadustat dosage based on HGB level if needed during the study. An online video and handbook will be provided to guide the data entry. The online forms will be provided for data validation to minimized errors with data entry. Data monitoring will be carried out to make sure the accuracy of the data entry. The main outcome of MACE will be judicated independently by two trained doctors using de-identified medical records. A third doctor will be consulted if there are any disagreements. The questionnaire on economic costs will be randomly selected and double-checked with medical insurance department to ensure the authenticity. The data will be locked once the data cleaning completes, and the analysis plan will be prepared and run independently by two statisticians.

\section{Data analysis}

The comparison in primary outcomes between the ROAD cohort and the registry data will be performed using Multiple Cox regression of risk factors and propensity score method will also be applied as a sensitivity analysis. The medical economic costs will be compared between ROAD cohort and registry control group using the whole population as well as populations stratified by hospital levels, regions, comorbidities.

The efficacy on HGB of Roxadustat in the treatment of anemia of the whole cohort as well as the pre-defined subgroups (including patients with evidence of erythropoietin resistance, patients with chronic inflammation, patients with impaired iron metabolism, and patients on hemodialysis or peritoneal dialysis) will be analyzed in intention-to-treat and per-protocol ROAD population. Treatment heterogeneity will be tested by adding an interaction term to the statistical models.

\section{Discussion}

Anemia is a common complication in patients with chronic kidney disease, especially in those on dialysis. Treatment of renal anemia is associated with improvement in cardiovascular function, and health related quality of life, reducing requirements for blood transfusion and hospitalization [18-20]. Erythropoiesis-stimulating agents are one of main treatments for anemia in dialysis [21]. Higher dosage of erythropoiesis-stimulating agents, however, is associated with risk of stroke [22-24], however, potential risks may occur according [25-28]. In addition, more than $10 \%$ patients were hyporesponsive to ESA $[29,30]$.

Roxadustat, a potent, reversible, HIF-PHI that mimics the natural response to hypoxia results in target genes involved in erythropoiesis [31-33]. The meta-analysis of randomized controlled trials showed that Roxadustat can improve hemoglobin levels and iron metabolism in both dialysis-dependent and non-dialysis-dependent chronic kidney disease [34, 35]. However, its long term efficacy and safety needs to be investigated.

This study is designed to systemically assess the impact of Roxadustat on dialysis patients in the real-world practice. The sites were chosen from different levels and different regions in the Sichuan province to make sure the repetitiveness of the study population. All treatment decision is made by the treating physician according to the patient's clinical conditions; however, the multidisciplinary team will provide consultation if required to improve the treatment of anemia. The data is collected both manually input and using software-based registry, 
which could increase the efficiency and reduce the typing errors. The software will be informative to assist doctors in treating dialysis patients with drug information, clinical guidelines and important alerts.

The main objective of the study is to provide the longterm impact of Roxadustat on MACE and all-cause mortality, economic burden, and safety. Differences in responding to Roxadustat in different subgroups will also be assessed. This study will be able to provide guidance in designing future, large scale randomized controlled trials assessing the impact of Roxadustat in dialysis patients.

\begin{abstract}
Abbreviations
REDCap: Research electronic data capture; ROAD: Roxadustat in treating anemia in dialysis patients; REACH: Renal disEAse observational CoHort database; CKD: Chronic kidney disease; ESRD: End-stage renal disease; EPO: Erythropoietin; ESA: Erythropoietin-stimulating agents; the DOPPS study: The Dialysis Outcomes and Practice Patterns Study; HGB: Hemoglobin; the CHOICE study: The Choices for Healthy Outcomes in Caring for ESRD study; HIF: Hypoxia inducible factor; HIF-PHI: Hypoxia inducible factor-prolyl hydroxylase inhibitor; MACE: Major adverse cardiovascular events; CKDMBD: Chronic kidney disease-mineral bone disorder; AE: Adverse events
\end{abstract}

\section{Acknowledgements}

We gratefully acknowledge the clinical research coordinators for their valuable comments on this study.

\section{Authors' contributions}

D.H. designed the study, G.L. and Q.H. supervised the study, Y.Z.1, S.R and Y.Z.2 drafted the manuscript, A.W. and X.W. provided critical review, H.X., Y.C., J.H., X.Y., F.J., J.W., D.Y., D.H., S.H., M.L., F.D. and J.C. collected data. The author(s) read and approved the final manuscript.

\section{Funding}

The work was supported by research grants from Department of Science and Technology of Sichuan Province No. 2019JDPT0007.

\section{Availability of data and materials}

Not applicable at this stage. The datasets analyzed during the current study will be available from the corresponding author on reasonable request when the study is finished.

\section{Ethics approval and consent to participate}

The study was approved by the Institutional Review Board at Sichuan Provincial People's Hospital (2019-196). The informed consent obtained from study participants will be written.

\section{Consent for publication}

Not Applicable.

\section{Competing interests}

The authors declare that they have no competing interests.

\section{Author details}

${ }^{1}$ Renal Department and Nephrology Institute, Sichuan Provincial People's Hospital, School of Medicine, University of Electronic Science and Technology of China, No. 32, West 2nd Duan, 1st Circle Road, Qingyang District, Chengdu, Sichuan, China. ${ }^{2}$ Department of Nephrology, Ya'an People's Hospital, 625000 Ya'an, Sichuan, China. ${ }^{3}$ The Renal and Metabolic Division, The George Institute for Global Health, University of New South Wales, Sydney 2042, Australia. ${ }^{4}$ Concord Clinical School, The University of Sydney, Sydney 2042, Australia. ${ }^{5}$ Department of Renal Medicine, Concord Repatriation General Hospital, Beijing Friendship Hospital, Beijing, China. ${ }^{6}$ Department of Nephrology, 610000 Gao Xin Boli Hospital,Chengdu, China. ${ }^{7}$ Department of Nephrology, The Second Affiliated Hospital of Chengdu
}

Medical College, National Nuclear Corporation 416 Hospital, 610000 Chengdu, China. ${ }^{8}$ Department of Nephrology, Sichuan Science City Hospital, 621000 Mianyang, China. ${ }^{9}$ Department of Nephrology, Chengdu Jinniu District People's Hospital, 610036 Chengdu, China. ${ }^{10}$ Hemodialysis center,Pidu District People's Hospital, 611730 Chengdu, China. ${ }^{11}$ Department of Nephrology, Mianyang Anzhou People's Hospital, 621000 Mianyang, China.

${ }^{12}$ Department of Nephrology, The People's Hospital of Mianyang, 621000 Mianyang, China. ${ }^{13}$ Department of Nephrology, Sichuan Mianyang 404 Hospital, 621000 Mianyang, China. ${ }^{14}$ Department of Nephrology, Affiliated Hospital of Chengdu University, 610081 Chengdu, China. ${ }^{15}$ The George Institute for Global Health, Faculty of Medicine, University of New South Wales, Sydney, NSW, Australia.

Received: 15 September 2020 Accepted: 1 January 2021

Published online: 13 January 2021

\section{References}

1. Borut C, Gitanjali PSA, Steven P. Z, R. CA. An Exploratory Study of Daprodustat in Erythropoietin-Hyporesponsive Subjects. Kidney International Reports. 2018:S2468024918300408-.

2. Zhang L, Fang W, Li W, et al. Prevalence of chronic kidney disease in China: a cross-sectional survey. Lancet. 2012;379(9818):0-822.

3. T O, M TA R, M U. Intermittent Oral Dosing of Roxadustat in Peritoneal Dialysis Chronic Kidney Disease Patients with Anemia: A Randomized, Phase 3, Multicenter, Open-Label Study. Therapeutic apheresis and dialysis: official peer-reviewed journal of the International Society for Apheresis, the Japanese Society for Apheresis, the Japanese Society for Dialysis Therapy. 2019.

4. Nakhoul G, Simon JF. Anemia of chronic kidney disease: Treat it, but not too aggressively. Cleve Clin J Med. 2016;83(8):613-24.

5. JENNIE Z. MA, Ebben J, XIA H, Collins AJ. Hematocrit Level and Associated Mortality in Hemodialysis Patients. Journal of the American Society of Nephrology 1999;10(3):610-9.

6. Walker MA. Anemia as a Predictor of Cardiovascular Events in Patients with Elevated Serum Creatinine. Journal of the American Society of Nephrology. 2006;17(8):2293-8.

7 Foley RN, Parfrey PS, Harnett JD, Kent GM, Murray DC, Barre PE. The impact of anemia on cardiomyopathy, morbidity, and mortality in end-stage renal disease. 1996;28(1):53-61.

8 Chen N, Hao C, Peng X, Lin H. Roxadustat for Anemia in Patients with Kidney Disease Not Receiving Dialysis. The New England Journal of Medicine. 2019;381:1001-10

9 Francesco L, Pisoni RL, Christian C, et al. Anaemia in haemodialysis patients of five European countries: association with morbidity and mortality in the Dialysis Outcomes and Practice Patterns Study (DOPPS). Nephrology Dialysis Transplantation. 2004;1:1.

10. SINGH A. Correction of anemia with epoetin alfa in chronic kidney disease. N Engl J Med. 2006;355:2085-98.

11. Chen N, Hao C, Liu B-C, Lin H, Wang C. X C. Roxadustat Treatment for Anemia in Patients Undergoing Long-Term Dialysis. The New England Journal of Medicine. 2019;12(381):1011-22.

12. Akizawa T, Iwasaki M, Otsuka T. Roxadustat Treatment of Chronic Kidney DiseaseAssociated Anemia in Japanese Patients Not on Dialysis: A Phase 2, Randomized, Double-Blind, Placebo-Controlled Trial. Advances in Therapy. 2019;36:1438-54.

13 Anatole B, Robert $\mathrm{P}$, Joachim $\mathrm{H}$, et al. Randomized placebo-controlled doseranging and pharmacodynamics study of roxadustat (FG-4592) to treat anemia in nondialysis-dependent chronic kidney disease (NDD-CKD) patients. Nephrology Dialysis Transplantation. 2015;10:10.

14. Harris PA, Taylor R, Thielke R, Payne J, Gonzalez N, Conde JG. Research electronic data capture (REDCap)--a metadata-driven methodology and workflow process for providing translational research informatics support. J Biomed Inform. 2009;42(2):377-81.

15. Harris PA, Taylor R, Minor BL, et al. The REDCap consortium: Building an international community of software platform partners. J Biomed Inform. 2019:95:103208

16. Locatelli F, Hannedouche T, Fishbane S, Morgan Z, Oguey D, White WB. Cardiovascular Safety and All-Cause Mortality of Methoxy Polyethylene Glycol-Epoetin Beta and Other Erythropoiesis-Stimulating Agents in Anemia of CKD: A Randomized Noninferiority Trial. Clinical journal of the American Society of Nephrology: CJASN. 2019;14(12):1701-10. 
17. Mc Causland FR, Claggett B, Burdmann EA, et al. Treatment of Anemia With Darbepoetin Prior to Dialysis Initiation and Clinical Outcomes: Analyses From the Trial to Reduce Cardiovascular Events With Aranesp Therapy (TREA T). American journal of kidney diseases: the official journal of the National Kidney Foundation. 2019;73(3):309-15.

18. Spinowitz B, Pecoits-Filho R, Winkelmayer WC, et al. Economic and quality of life burden of anemia on patients with CKD on dialysis: a systematic review. J Med Econ. 2019;22(6):593-604.

19. Foley RN, Curtis BM, Parfrey PS. Erythropoietin therapy, hemoglobin targets, and quality of life in healthy hemodialysis patients: a randomized trial. Clinical journal of the American Society of Nephrology: CJASN. 2009;4(4): 726-33.

20. Foley RN, Curtis BM, Parfrey PS. Hemoglobin targets and blood transfusions in hemodialysis patients without symptomatic cardiac disease receiving erythropoietin therapy. Clinical journal of the American Society of Nephrology: CJASN. 2008;3(6):1669-75.

21. Akizawa T, Okumura H, Alexandre AF, Fukushima A, Kiyabu G, Dorey J. Burden of Anemia in Chronic Kidney Disease Patients in Japan: A Literature Review. Therapeutic apheresis dialysis: official peer-reviewed journal of the International Society for Apheresis the Japanese Society for Apheresis the Japanese Society for Dialysis Therapy. 2018;22(5):444-56.

22. Drueke TB, Locatelli F, Clyne N, et al. Normalization of hemoglobin level in patients with chronic kidney disease and anemia. The New England journal of medicine. 2006;355(20):2071-84.

23. Singh AK, Szczech L, Tang KL, et al. Correction of anemia with epoetin alfa in chronic kidney disease. The New England journal of medicine. 2006; 355(20):2085-98

24. Macdougall IC, White C, Anker SD, et al. Intravenous Iron in Patients Undergoing Maintenance Hemodialysis. The New England journal of medicine. 2019;380(5):447-58.

25. Besarab A, Bolton WK, Browne JK, et al. The effects of normal as compared with low hematocrit values in patients with cardiac disease who are receiving hemodialysis and epoetin. The New England journal of medicine. 1998;339(9):584-90.

26. Solomon SD, Uno H, Lewis EF, et al. Erythropoietic response and outcomes in kidney disease and type 2 diabetes. The New England journal of medicine. 2010;363(12):1146-55.

27. Szczech $L A$, Barnhart $H X$, Inrig JK, et al. Secondary analysis of the CHOIR trial epoetin-alpha dose and achieved hemoglobin outcomes. Kidney international. 2008;74(6):791-8.

28. Canavese C, Bergamo D, Ciccone $G$, et al. Validation of serum ferritin values by magnetic susceptometry in predicting iron overload in dialysis patients. Kidney international. 2004;65(3):1091-8.

29. Luo J, Jensen DE, Maroni BJ, Brunelli SM. Spectrum and Burden of Erythropoiesis-Stimulating Agent Hyporesponsiveness Among Contemporary Hemodialysis Patients. American journal of kidney diseases: the official journal of the National Kidney Foundation. 2016;68(5):763-71.

30. Malyszko J, Malyszko JS, Mysliwiec M. Hyporesponsiveness to erythropoietin therapy in hemodialyzed patients: potential role of prohepcidin, hepcidin, and inflammation. Renal failure. 2009;31(7):544-8.

31. Peyssonnaux C, Nizet V, Johnson RS. Role of the hypoxia inducible factors HIF in iron metabolism. Cell Cycle. 2008;7(1):28-32.

32. Wang $G L$, Jiang $B H$, Rue EA, Semenza GL. Hypoxia-inducible factor 1 is a basic-helix-loop-helix-PAS heterodimer regulated by cellular $\mathrm{O} 2$ tension. Proc Natl Acad Sci U S A. 1995;92(12):5510-4.

33. Semenza GL. Hypoxia-inducible factor 1: master regulator of $\mathrm{O} 2$ homeostasis. Curr Opin Genet Dev. 1998;8(5):588-94.

34. Jia L, Dong X, Yang J, Jia R, Zhang H. Effectiveness of hypoxia-inducible factor prolyl hydroxylase inhibitor roxadustat on renal anemia in nondialysis-dependent chronic kidney disease: a systematic review and metaanalysis. Ann Transl Med. 2019;7(23):720

35. Liu J, Zhang A, Hayden JC, et al. Roxadustat (FG-4592) treatment for anemia in dialysis-dependent (DD) and not dialysis-dependent (NDD) chronic kidney disease patients: A systematic review and meta-analysis. Pharmacol Res. 2020;155:104747.

\section{Publisher's Note}

Springer Nature remains neutral with regard to jurisdictional claims in published maps and institutional affiliations.

Ready to submit your research? Choose BMC and benefit from:

- fast, convenient online submission

- thorough peer review by experienced researchers in your field

- rapid publication on acceptance

- support for research data, including large and complex data types

- gold Open Access which fosters wider collaboration and increased citations

- maximum visibility for your research: over $100 \mathrm{M}$ website views per year

At $\mathrm{BMC}$, research is always in progress.

Learn more biomedcentral.com/submissions 